\title{
Triezenberg-Zwanzig expression for the surface tension of a liquid drop
}

Cite as: J. Chem. Phys. 138, 194711 (2013); https://doi.org/10.1063/1.4805001

Submitted: 27 February 2013 . Accepted: 30 April 2013 . Published Online: 21 May 2013

\section{Edgar M. Blokhuis}

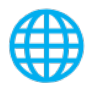

\section{ARTICLES YOU MAY BE INTERESTED IN}

Surface thermodynamics of planar, cylindrical, and spherical vapour-liquid interfaces of water The Journal of Chemical Physics 142, 114701 (2015); https://doi.org/10.1063/1.4913371

The tension of a curved surface from simulation

The Journal of Chemical Physics 137, 234101 (2012); https://doi.org/10.1063/1.4769880

A molecular dynamics study of liquid drops

The Journal of Chemical Physics 81, 530 (1984); https://doi.org/10.1063/1.447358

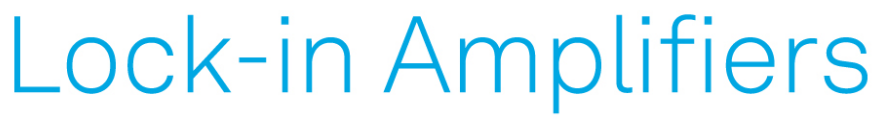
... and more, from DC to $600 \mathrm{MHz}$

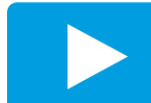

Watch 


\title{
Triezenberg-Zwanzig expression for the surface tension of a liquid drop
}

\author{
Edgar M. Blokhuis ${ }^{a)}$ \\ Colloid and Interface Science, Leiden Institute of Chemistry, Gorlaeus Laboratories, P.O. Box 9502, \\ 2300 RA Leiden, The Netherlands
}

(Received 27 February 2013; accepted 30 April 2013; published online 21 May 2013)

\begin{abstract}
Formulas, analogous to the Triezenberg-Zwanzig expression for the surface tension of a planar interface, are presented for the Tolman length, the bending rigidity, and the rigidity constant associated with Gaussian curvature. These expressions feature the Ornstein-Zernike direct correlation function and are derived from considering the deformation of a liquid drop in the presence of an external field. This approach is in line with the original analysis by Yvon [in Proceedings of the IUPAP Symposium on Thermodynamics, Brussels, 1948]. It is shown that our expressions reduce to previous results from density functional theory when a mean-field approximation is made for the direct correlation function. We stress the importance of the form of the external field used and show how the values of the rigidity constants depend on it. (C) 2013 AIP Publishing LLC. [http://dx.doi.org/10.1063/1.4805001]
\end{abstract}

\section{INTRODUCTION}

The most important quantity to define the properties of a liquid surface is its free energy or surface tension. The determination of the surface tension is therefore one of the key objectives in the Statistical Thermodynamical treatment of liquid surfaces. ${ }^{1}$ Over the years, different routes have led to different expressions for the surface tension each having their own merits and limitations.

At the start of any historical overview are mean-field expressions for the surface tension. At the beginning lies the squared-gradient expression derived by van der Waals in $1893:^{2}$

$$
\sigma=2 m \int_{-\infty}^{\infty} d z \rho_{0}^{\prime}(z)^{2}
$$

where $\rho_{0}(z)$ is the density profile of a planar interface. The squared-gradient expression is a particular result derived from the more general density functional theory (DFT), ${ }^{3-6}$ at the heart of which lies a division of the interaction potential in a hard sphere reference system perturbed by attractive forces, $U(r)=U_{\mathrm{hs}}(r)+U_{\mathrm{att}}(r)$. For the surface tension, DFT gives ${ }^{1}$

$$
\sigma=-\frac{1}{4} \int_{-\infty}^{\infty} d z_{1} \int d \vec{r}_{12} U_{\text {att }}(r) r^{2}\left(1-s^{2}\right) \rho_{0}^{\prime}\left(z_{1}\right) \rho_{0}^{\prime}\left(z_{2}\right)
$$

where the integration over the interparticle distance vector, $\vec{r}_{12} \equiv \vec{r}_{2}-\vec{r}_{1}$, is

$$
\int d \vec{r}_{12}=\int_{0}^{2 \pi} d \varphi \int_{-1}^{1} d s \int_{0}^{\infty} d r r^{2}
$$

which defines $s=\cos \left(\theta_{12}\right)$ and $z_{2}=z_{1}+r s$. A gradient expansion of the density in the expression for the surface tension

\footnotetext{
a)E-mail: e.blokhuis@chem.leidenuniv.nl
}

in Eq. (2) yields the van der Waals expression in Eq. (1) with the identification, $m=-(1 / 12) \int d \vec{r}_{12} r^{2} U_{\text {att }}(r)$.

A second approach results in the so-called virial expression for the surface tension, first derived by Kirkwood and Buff in 1948: ${ }^{7}$

$$
\sigma=\frac{1}{4} \int_{-\infty}^{\infty} d z_{1} \int d \vec{r}_{12} U^{\prime}(r) r\left(1-3 s^{2}\right) \rho_{0}^{(2)}\left(z_{1}, z_{2}, r\right)
$$

where $\rho_{0}^{(2)}\left(z_{1}, z_{2}, r\right)$ is the pair density correlation function of a planar interface. Even though this expression is derived by Kirkwood and Buff using a mechanical approach, by way of the evaluation of the components of the pressure tensor in the interfacial region, ${ }^{7}$ it is an exact equation, the only assumption being made is that of pair-wise additivity of the molecular interactions. The theoretical evaluation of the surface tension using the Kirkwood-Buff expression requires knowledge of the pair correlation function in the interfacial region, which is hard to access theoretically but can be determined to great accuracy in computer simulations. ${ }^{8,9}$

A third approach, which also provides an expression for the surface tension that is exact, results in the so-called Triezenberg-Zwanzig (TZ) expression: ${ }^{10,11}$

$\sigma=-\frac{k_{\mathrm{B}} T}{4} \int_{-\infty}^{\infty} d z_{1} \int d \vec{r}_{12} r^{2}\left(1-s^{2}\right) \rho_{0}^{\prime}\left(z_{1}\right) \rho_{0}^{\prime}\left(z_{2}\right) C_{0}\left(z_{1}, z_{2}, r\right)$,

where $C_{0}\left(z_{1}, z_{2}, r\right)$ is now the direct correlation function ${ }^{12}$ of the planar interface.

In this article, we are interested in deriving expressions for the surface tension of a curved surface. In particular, we investigate the expansion of the surface tension $\sigma(R)$ to second order in the (inverse) radius of curvature $R$ as described by the curvature coefficients $\delta$ (the Tolman length), ${ }^{13} k$ (the rigidity constant associated with bending or bending rigidity), and $\bar{k}$ (the rigidity constant associated with Gaussian curvature). For a spherical and cylindrical surface, this expansion 
has the form: ${ }^{14}$

$$
\begin{aligned}
& \sigma_{s}(R)=\sigma-\frac{2 \delta \sigma}{R}+\frac{(2 k+\bar{k})}{R^{2}}+\ldots \quad(\text { sphere) } \\
& \sigma_{c}(R)=\sigma-\frac{\delta \sigma}{R}+\frac{k}{2 R^{2}}+\ldots \quad \text { (cylinder). }
\end{aligned}
$$

The virial expressions and mean-field expressions (DFT) for $\delta, k$, and $\bar{k}$ have been derived some 20 years ago, ${ }^{15-17}$ but until now, the corresponding expressions in terms of the direct correlation function are lacking. Our goal in this article is to fill this void and to derive Triezenberg-Zwanzig-like expressions for the Tolman length $\delta$, and the rigidity constants $k$ and $\vec{k}$. To achieve this, we follow in the footsteps of the pioneering work by Jim Henderson and co-workers who addressed precisely this problem in three papers in the early 1980s. ${ }^{18-20}$ They derived an expression for the Tolman length in terms of the direct correlation function, which is consistent with the expression presented here. Furthermore, they pointed to difficulties in extending the analysis to second order in the expansion in the curvature, i.e., to the order of the rigidity constants $k$ and $\bar{k}$, which find their origin in the fact that the thermodynamic conditions used to impose a certain curvature of the interface then play a role.

The article is organized as follows. In Sec. II we address the thermodynamics involved in deriving expressions for the rigidity constants in general. Two distinct thermodynamic routes are formulated which correspond to the two original derivations of the Triezenberg-Zwanzig expression for the surface tension of the planar interface: (i) the fluctuation route, used by Triezenberg and Zwanzig themselves in 1972, in which one considers the second order change in free energy due to surface fluctuations; (ii) the equilibrium route, used by Lovett et al. ${ }^{21}$ following an approach suggested by Yvon in 1948, ${ }^{10}$ in which one investigates the deformation of a planar interface induced by an external field. These two approaches, which result in the same expression for the surface tension of the planar interface, are discussed in Sec. III and extended to curved surfaces. The full calculation of the Tolman length $\delta$ and the rigidity constants $k$ and $\bar{k}$ is presented in Sec. IV, with details of the calculations left to Appendices A-D. We end with a discussion of results.

\section{FLUCTUATION ROUTE VERSUS EQUILIBRIUM ROUTE}

Historically, the Triezenberg-Zwanzig expression for the surface tension has been derived in two distinct ways: (i) by the analysis of the second order change in free energy due to surface fluctuations; ${ }^{11}$ (ii) by applying an external field to deform an initially planar interface. ${ }^{10,21}$ These two routes, which we have termed previously ${ }^{22,23}$ as the fluctuation route and the equilibrium route, are connected to the (thermodynamic) path considered to deform the surface and to induce a certain interfacial curvature.

In the fluctuation route, the curvature of the interface naturally comes about by the presence of thermal fluctuations. The bulk region is unaffected and the chemical potential remains that at two-phase coexistence, $\mu=\mu_{\text {coex }}$. This route is in many senses equivalent to a route in which one considers the presence of a non-uniform external field that is non-zero in the interfacial region only. The "fluctuation" is then envisioned as being induced by the non-uniform external field.

In the equilibrium route, the interface is curved by changing the value of the chemical potential to a value offcoexistence at fixed temperature $\left(\mu>\mu_{\text {coex }}\right)$. One then considers the surface tension $\sigma(R)$ of a spherically (or cylindrically) shaped liquid droplet with radius $R$ in metastable equilibrium with a bulk vapour. In effect, a uniform external field is added of the form $V_{\text {ext }}(\vec{r})=-\Delta \mu$, where $\Delta \mu=\mu-\mu_{\text {coex }}$. Since the uniform external field acts throughout the system, it also affects the bulk densities far from the interfacial region.

These two distinct routes lead to the same expression for the planar surface tension, but it was already noted by Henderson and co-workers ${ }^{18-20}$ that this may not be the case for the radius dependent surface tension $\sigma(R)$, and in particular for the coefficients in an expansion in $1 / R$ of the surface tension beyond the Tolman length. Their concerns were connected to the fact that different external fields may lead to the same interfacial curvature and that it is not a priori clear that the expression for the radius dependent surface tension derived using one external field equals that of another. Although the two routes $d o$ lead to the same expression for the surface tension, it turns out that this indeed is not the case for the rigidity constants. This was demonstrated previously in the context of DFT expressions for the bending rigidity $k:^{23,24}$ the fluctuation route was investigated by supposing the presence of an external field chosen proportional to the derivative of the density profile to ensure that it acts in the interfacial region only. ${ }^{23}$ It was shown that the value obtained for the bending rigidity then differs in magnitude and scaling behaviour from that obtained using the equilibrium route ${ }^{24}$ (see Appendix A).

That the thermodynamic conditions imposed to induce a certain interfacial curvature have bearing on the rigidity constants, may also be deduced from the fact that the rigidity constants depend on the density profile $\rho_{1}(z)$, which describes the leading order change in the distribution of molecules when the interface is curved. The fact that the density profiles $\rho_{1}(z)$ are different in the fluctuation and equilibrium route, follows directly from the observation that in the equilibrium route, $\rho_{1}(z)$ is not equal to zero in the bulk regions since the chemical potential is also different $\left(\mu \neq \mu_{\text {coex }}\right)$, while it is zero in the fluctuation route since fluctuations of the interface do not affect the thermodynamic state of the bulk regions.

With this important observation in mind, we consider, in Sec. III the usual derivations of the Triezenberg-Zwanzig expression for the surface tension of a planar interface and discuss how they are extended to determine the Tolman length $\delta$ and the rigidity constants $k$ and $\bar{k}$.

\section{TWO DERIVATIONS OF THE TRIEZENBERG-ZWANZIG EXPRESSION FOR THE SURFACE TENSION}

To understand how the direct correlation function enters in the Triezenberg-Zwanzig expression for the surface tension, we need to address the Statistical Thermodynamical definition of the direct correlation function. ${ }^{12}$ 
In the fluctuation route considered first, the direct correlation function describes the (second order) response of the free energy due to density fluctuations. This route was used in the original derivation by Triezenberg and Zwanzig ${ }^{11}$ in 1972 . In the equilibrium route, the direct correlation function describes how a small change in the local density can be interpreted as a change in the local external field (second Yvon equation). This approach, inspired by the work of Yvon in 1948, ${ }^{10}$ was used by Lovett et $a .^{21}$ to rederive the TZ expression for the surface tension of the planar interface by considering the external field necessary to bend an initially flat surface into a spherical droplet. This procedure was then extended by Henderson and Schofield ${ }^{19}$ to spherical droplets in order to have access to the full radius dependent surface tension.

\section{A. Fluctuation route}

In the fluctuation route, one investigates the change in free energy due to density fluctuations around a planar interface. The direct correlation function describes the second order change in the free energy in response to such a density fluctuation:

$$
\Delta \Omega=\frac{k_{\mathrm{B}} T}{2} \int d \vec{r}_{1} \int d \vec{r}_{12} C_{0}\left(z_{1}, z_{2}, r\right) \delta \rho\left(\vec{r}_{1}\right) \delta \rho\left(\vec{r}_{2}\right) .
$$

The density fluctuation can be expressed in terms of a height function $h\left(\vec{r}_{\|}\right)$that describes the shift in the location of the surface: ${ }^{23,25}$

$$
\delta \rho(\vec{r}) \equiv \rho(\vec{r})-\rho_{0}(z)=-\rho_{0}^{\prime}(z) h\left(\vec{r}_{\|}\right)-\frac{\rho_{1}(z)}{2} \vec{\nabla}^{2} h\left(\vec{r}_{\|}\right)+\ldots,
$$

where it is assumed that $|\vec{\nabla} h| \ll 1$ and where $\rho_{1}(z)$ is identified as the correction to the density profile due to the curvature of the interface. In general, $\rho_{1}(z)$ describes the rearrangement of molecules that results when the interface is curved, but its form still depends on the way the curvature is brought upon.

The surface tension and bending rigidity are thermodynamically defined as the change in free energy due to height fluctuations: ${ }^{26}$

$$
\Delta \Omega=\frac{1}{2} \int \frac{d \vec{q}}{(2 \pi)^{2}}\left[\sigma q^{2}+k q^{4}+\ldots\right] h(\vec{q}) h(-\vec{q}) .
$$

Inserting Eq. (8) into Eq. (7) and comparing the result to Eq. (9), we retrieve the TZ expression for the surface tension in Eq. (5). A further result is that for the bending rigidity one finds: ${ }^{25}$

$$
\begin{aligned}
k= & \frac{k_{\mathrm{B}} T}{4} \int_{-\infty}^{\infty} d z_{1} \int d \vec{r}_{12} \rho_{1}\left(z_{1}\right) \rho_{1}\left(z_{2}\right) C_{0}\left(z_{1}, z_{2}, r\right) \\
& +\frac{k_{\mathrm{B}} T}{4} \int_{-\infty}^{\infty} d z_{1} \int d \vec{r}_{12} r^{2}\left(1-s^{2}\right) \rho_{0}^{\prime}\left(z_{1}\right) \rho_{1}\left(z_{2}\right) C_{0}\left(z_{1}, z_{2}, r\right) \\
& +\frac{k_{\mathrm{B}} T}{64} \int_{-\infty}^{\infty} d z_{1} \int d \vec{r}_{12} r^{4}\left(1-s^{2}\right)^{2} \rho_{0}^{\prime}\left(z_{1}\right) \rho_{0}^{\prime}\left(z_{2}\right) C_{0}\left(z_{1}, z_{2}, r\right) .
\end{aligned}
$$

This formal expression for $k$ was first derived by Parry and Boulter. ${ }^{25}$ Even though this expression is exact, for the explicit evaluation of it, one still requires some way of determining the density profile $\rho_{1}(z)$. Furthermore, expressions for $\delta$ and $\bar{k}$ cannot be derived using this route.

\section{B. Equilibrium route}

To understand the way the direct correlation function enters the expression for the surface tension in the equilibrium route, we need to discuss the two Yvon equations which are in the heart of any Statistical Thermodynamics treatment of surfaces. ${ }^{1,4,5}$ In the first Yvon equation, the total correlation function, $G\left(\vec{r}_{1}, \vec{r}_{2}\right)$, relates a small change in the local external field to a change in the local density: ${ }^{4,5}$

$$
\delta \rho\left(\vec{r}_{1}\right)=-\frac{1}{k_{\mathrm{B}} T} \int d \vec{r}_{2} G\left(\vec{r}_{1}, \vec{r}_{2}\right) \delta V_{\mathrm{ext}}\left(\vec{r}_{2}\right),
$$

where $G\left(\vec{r}_{1}, \vec{r}_{2}\right)$ is related to the pair density as

$$
G\left(\vec{r}_{1}, \vec{r}_{2}\right)=\rho^{(2)}\left(\vec{r}_{1}, \vec{r}_{2}\right)-\rho\left(\vec{r}_{1}\right) \rho\left(\vec{r}_{2}\right)+\rho\left(\vec{r}_{1}\right) \delta\left(\vec{r}_{2}-\vec{r}_{1}\right) .
$$

The inverse of the total correlation function defines the $d i$ rect correlation function ${ }^{12}$ and it describes in the second Yvon equation how a small change in the local density can be attributed to a change in the local external field: ${ }^{4,5}$

$$
\delta V_{\mathrm{ext}}\left(\vec{r}_{1}\right)=-k_{\mathrm{B}} T \int d \vec{r}_{2} C\left(\vec{r}_{1}, \vec{r}_{2}\right) \delta \rho\left(\vec{r}_{2}\right) .
$$

By considering translational deformations, the second Yvon equation appears in the following form:

$$
\vec{\nabla}_{1} V_{\text {ext }}\left(\vec{r}_{1}\right)=-k_{\mathrm{B}} T \int d \vec{r}_{2} C\left(\vec{r}_{1}, \vec{r}_{2}\right) \vec{\nabla}_{2} \rho\left(\vec{r}_{2}\right),
$$

which, in planar geometry, reduces to

$$
V_{\mathrm{ext}}^{\prime}\left(z_{1}\right)=-k_{\mathrm{B}} T \int d \vec{r}_{2} C_{0}\left(z_{1}, z_{2}, r\right) \rho_{0}^{\prime}\left(z_{2}\right) .
$$

As a next step, we consider the influence of the external field on the Laplace pressure difference $\Delta p$ across a spherical or cylindrical droplet with radius $R$. The generalized Laplace equation for the pressure difference across a surface with (constant) curvatures $J=1 / R_{1}+1 / R_{2}$ and $K=1 /\left(R_{1} R_{2}\right)$ is given by ${ }^{27}$

$$
\Delta p=\sigma J-2 \delta \sigma K-\frac{k}{2} J^{3}+2 k J K+\ldots
$$

For a spherically or cylindrically shaped surface, this expansion takes the form:

$$
\begin{aligned}
& \Delta p=\frac{2 \sigma}{R}-\frac{2 \delta \sigma}{R^{2}}+\ldots \quad(\text { sphere }), \\
& \Delta p=\frac{\sigma}{R}-\frac{k}{2 R^{3}}+\ldots \quad \text { (cylinder) }
\end{aligned}
$$

where the dots represent terms of order $\mathcal{O}\left(1 / R^{4}\right)$, which indicates that the term proportional to $1 / R^{3}$ is absent in the expansion of the spherical interface. It is important to mention that these expressions are derived with the equimolar radius ${ }^{28}$ chosen as the radius of the spherically (or cylindrically) shaped 
surface, i.e., $R=R_{e}$, where $R_{e}$ for a spherical liquid droplet is defined by

$$
4 \pi \int_{0}^{\infty} d r r^{2}\left[\rho_{s}(r)-\rho_{v}\right]=\frac{4 \pi}{3} R_{e}^{3}\left(\rho_{\ell}-\rho_{v}\right)
$$

Next, we consider the consequences of mechanical balance in the presence of an external field. The condition of mechanical balance can be expressed in terms of the pressure tensor: ${ }^{1}$

$$
\vec{\nabla} \cdot \overrightarrow{\vec{p}}(\vec{r})=-\rho(\vec{r}) \vec{\nabla} V_{\text {ext }}(\vec{r}) .
$$

In spherical symmetry, the expression for mechanical balance leads to

$$
p_{\mathrm{N}}^{\prime}(r)=\frac{2}{r}\left[p_{\mathrm{T}}(r)-p_{\mathrm{N}}(r)\right]-\rho_{s}(r) V_{\mathrm{ext}}^{\prime}(r),
$$

which integrated from inside the liquid to the vapour phase gives

$$
\Delta p=\int_{0}^{\infty} d r \frac{2}{r}\left[p_{\mathrm{N}}(r)-p_{\mathrm{T}}(r)\right]+\int_{0}^{\infty} d r \rho_{s}(r) V_{\mathrm{ext}}^{\prime}(r) .
$$

Following the derivation by Yvon, ${ }^{10}$ this expression was applied by Lovett et al. $^{21}$ to rederive the TZ expression for the surface tension by considering the situation in which an initially flat surface, in the absence of an external field, is bend into a spherical droplet by an infinitesimally small external field $\delta V_{\text {ext }}(r)$ in such a way that the bulk regions are unaffected. More generally, one may consider an already curved spherical droplet, whose curvature is increased or decreased $(R \rightarrow R+\delta R)$ by the infinitesimally small external field. ${ }^{19}$ In either case, the change in pressure difference is zero, $\delta(\Delta p)=$ 0 , and the two terms on the right-hand-side of Eq. (22) must balance. Since we have that in the absence of the external field

$$
\Delta p=\int_{0}^{\infty} d r \frac{2}{r}\left[p_{\mathrm{N}}(r)-p_{\mathrm{T}}(r)\right]=\frac{2 \sigma}{R}-\frac{2 \delta \sigma}{R^{2}}+\ldots,
$$

this implies that

$$
\delta\left(\frac{2 \sigma}{R}-\frac{2 \delta \sigma}{R^{2}}+\ldots\right)=\int_{0}^{\infty} d r \rho_{s}^{\prime}(r) \delta V_{\mathrm{ext}}(r),
$$

where we have integrated the last term in Eq. (22) by parts. The second Yvon equation in Eq. (13) is then used to rewrite the right-hand-side as

$$
\begin{aligned}
& \left(\frac{2 \sigma}{R^{2}}-\frac{4 \delta \sigma}{R^{3}}+\ldots\right) \delta R \\
& \quad=k_{\mathrm{B}} T \int_{0}^{\infty} d r_{1} \int d \vec{r}_{2} C_{s}\left(r_{1}, r_{2}, r\right) \rho_{s}^{\prime}\left(r_{1}\right) \delta \rho_{s}\left(r_{2}\right) .
\end{aligned}
$$

The leading order term in the expansion in $1 / R$ of the righthand-side in this equation leads to the TZ expression for $\sigma,{ }^{21}$ while the next to leading order term leads to an expression for the Tolman length. ${ }^{18,19}$ Here, we are interested in the second order terms beyond the leading term. Even though the coefficient of the $1 / R^{4}$ term in Eq. (25) happens to be zero, its absence shall provide information for the determination of the combination of rigidity constants $2 k+\bar{k}$.

In order to obtain an expression for the bending rigidity $k$ separately, the analysis is also carried out for a cylindrically shaped interface. In cylindrical geometry, the consideration of mechanical balance than ultimately leads to

$$
\begin{aligned}
& \left(\frac{\sigma}{R^{2}}-\frac{3 k}{2 R^{4}}+\ldots\right) \delta R \\
& \quad=k_{\mathrm{B}} T \int_{0}^{\infty} d r_{1} \int d \vec{r}_{2} C_{c}\left(r_{1}, r_{2}, \varphi, r\right) \rho_{c}^{\prime}\left(r_{1}\right) \delta \rho_{c}\left(r_{2}\right) .
\end{aligned}
$$

For the cylindrical interface, the $1 / R^{4}$ term is related to the bending rigidity $k$ and it is the $1 / R^{3}$ term that vanishes. In Sec. IV, Eqs. (25) and (26) are used to carry out a systematic expansion in $1 / R$ to derive explicit expressions for the Tolman length $\delta$, and the rigidity constants $k$ and $\bar{k}$.

\section{EQUILIBRIUM ROUTE TO DETERMINE $\delta, k$, AND $\bar{k}$}

We consider the situation outlined in Sec. III: a spherical liquid droplet with radius $R$ is deformed by an infinitesimally small external field into another spherical droplet with radius $R \rightarrow R+\delta R$ without affecting the bulk regions. To determine the resulting change in density profile, we use the fact that we can write the density as $\rho_{s}(r)=\rho_{s}(r-R ; R)$. This means that when $R$ is changed, the density profile is affected in two ways: (i) the variable $z \equiv r-R$ is shifted and (ii) the density profile itself depends on $R$. This means that

$$
\begin{aligned}
\delta \rho_{s}(r) & =\rho_{s}(r-R-\delta R ; R+\delta R)-\rho_{s}(r-R ; R) \\
& =-\rho_{s}^{\prime}(r) \delta R+\rho_{s}(r ; R+\delta R)-\rho_{s}(r ; R) .
\end{aligned}
$$

When we expand $\rho_{s}(r)$ in $1 / R$, as in Eq. (A3), this gives

$$
\delta \rho_{s}(r)=-\left[\rho_{s}^{\prime}(r)+\frac{\rho_{1}(r)}{R^{2}}+\frac{2 \rho_{s, 2}(r)}{R^{3}}+\ldots\right] \delta R .
$$

This expression is inserted into Eq. (25) and expanded to second order beyond the leading order term. Details of the analysis for spherical droplets are outlined in Appendix B; details of the analysis for cylindrical droplets are given in Appendix C. For the Tolman length, the analysis of the spherical interface in Appendix B leads to Eq. (B9):

$$
\delta \sigma=\frac{k_{\mathrm{B}} T}{8} \int_{-\infty}^{\infty} d z_{1} \int d \vec{r}_{12} r^{2}\left(1-s^{2}\right)\left[\rho_{s}^{\prime}\left(z_{1}\right) \rho_{s}^{\prime}\left(z_{2}\right) C_{s}\left(z_{1}, z_{2}, r\right)\right]_{1},
$$

where the subscript 1 refers to the leading order correction in an expansion in $1 / R$ (see Eq. (A3)). The evaluation of the Tolman length using this expression therefore requires knowledge of the way the density profile and direct correlation function depend on the radius of curvature.

The expression for the Tolman length in Eq. (29) can also be derived from the expression for $\sigma_{s}(R)$ presented by 
Henderson and co-workers in Refs. 18 and 19:

$$
\begin{aligned}
\sigma_{s}(R)= & -\frac{k_{\mathrm{B}} T}{4} \int_{0}^{\infty} d r_{1} \int d \vec{r}_{12}\left[r^{2}-\left(r_{1}-r_{2}\right)^{2}\right] \\
& \times \rho_{s}^{\prime}\left(r_{1}\right) \rho_{s}^{\prime}\left(r_{2}\right) C_{s}\left(r_{1}, r_{2}, r\right),
\end{aligned}
$$

which is constructed to be correct to order $\mathcal{O}(1 / R) .{ }^{19}$ An explicit expansion in $1 / R$ of the term in square brackets gives

$$
r^{2}-\left(r_{1}-r_{2}\right)^{2}=r^{2}\left(1-s^{2}\right)\left(1-\frac{s r}{R}\right)+\ldots
$$

Inserting this expansion in Eq. (30) and comparing with the expansion for $\sigma_{s}(R)$ in Eq. (6) results in the expression for the Tolman length in Eq. (29). It should be mentioned that starting with the Henderson expression in Eq. (30), an expression for the Tolman length very similar to Eq. (29) was derived by Barrett in 1999. ${ }^{29}$ The only difference is that in the Barrett expression for the Tolman length, $C_{s, 1}\left(z_{1}, z_{2}, r\right)$ in Eq. (29) is rewritten in terms of the triplet direct correlation function of the planar interface. ${ }^{29}$

When the expression for the Tolman length in Eq. (29) is combined with the result of the analysis of the cylindrical interface in Appendix $\mathrm{C}$, we find as an alternative expression for the Tolman length:

$\delta \sigma=\frac{k_{\mathrm{B}} T}{4} \int_{-\infty}^{\infty} d z_{1} \int d \vec{r}_{12} r^{2}\left(1-s^{2}\right) z_{1} \rho_{0}^{\prime}\left(z_{1}\right) \rho_{0}^{\prime}\left(z_{2}\right) C_{0}\left(z_{1}, z_{2}, r\right)$.

It is reminded that in this expression the location of the $z$ $=0$ plane corresponds to the equimolar surface. The advantage of this expression for the Tolman length is that it can be evaluated from the properties of the planar interface only. In Appendix A we verify that both expressions for the Tolman length in Eqs. (29) and (32) are consistent with known DFT expressions. $^{17,24}$

The vanishing of the $1 / R^{4}$ term in Eq. (25) in the analysis for the spherical interface, leads to Eq. (B10) in Appendix B. This gives the following two expressions for the combination $2 k+\bar{k}$ :

$$
\begin{aligned}
2 k+\bar{k}= & -\frac{k_{\mathrm{B}} T}{12} \int_{-\infty}^{\infty} d z_{1} \int d \vec{r}_{12} r^{2}\left(1-s^{2}\right)\left[\rho_{s}^{\prime}\left(z_{1}\right) \rho_{s}^{\prime}\left(z_{2}\right)\right. \\
& \left.\times C_{s}\left(z_{1}, z_{2}, r\right)\right]_{2} \\
& -\frac{2 k_{\mathrm{B}} T}{3} \int_{-\infty}^{\infty} d z_{1} \int d \vec{r}_{12} r s \rho_{0}^{\prime}\left(z_{1}\right) \rho_{s, 2}\left(z_{2}\right) C_{0}\left(z_{1}, z_{2}, r\right) \\
& -\frac{k_{\mathrm{B}} T}{3} \int_{-\infty}^{\infty} d z_{1} \int d \vec{r}_{12} r s \rho_{1}\left(z_{2}\right)\left[\rho_{s}^{\prime}\left(z_{1}\right) C_{s}\left(z_{1}, z_{2}, r\right)\right]_{1} \\
& -\frac{k_{\mathrm{B}} T}{4} \int_{-\infty}^{\infty} d z_{1} \int d \vec{r}_{12} r^{2}\left(1-s^{2}\right) z_{1}^{2} \rho_{0}^{\prime}\left(z_{1}\right) \rho_{0}^{\prime}\left(z_{2}\right) \\
& \times C_{0}\left(z_{1}, z_{2}, r\right)
\end{aligned}
$$

$$
\begin{aligned}
& +\frac{k_{\mathrm{B}} T}{48} \int_{-\infty}^{\infty} d z_{1} \int d \vec{r}_{12} r^{4}\left(1-s^{4}\right) \rho_{0}^{\prime}\left(z_{1}\right) \rho_{0}^{\prime}\left(z_{2}\right) \\
& \times C_{0}\left(z_{1}, z_{2}, r\right)
\end{aligned}
$$

and

$$
\begin{aligned}
2 k+\bar{k}= & -\frac{k_{\mathrm{B}} T}{6} \int_{-\infty}^{\infty} d z_{1} \int d \vec{r}_{12} r^{2}\left(1-s^{2}\right) z_{1}\left[\rho_{s}^{\prime}\left(z_{1}\right) \rho_{s}^{\prime}\left(z_{2}\right)\right. \\
& \left.\times C_{s}\left(z_{1}, z_{2}, r\right)\right]_{1} \\
& -\frac{k_{\mathrm{B}} T}{3} \int_{-\infty}^{\infty} d z_{1} \int d \vec{r}_{12} r s z_{1} \rho_{0}^{\prime}\left(z_{1}\right) \rho_{1}\left(z_{2}\right) C_{0}\left(z_{1}, z_{2}, r\right) \\
& +\frac{k_{\mathrm{B}} T}{12} \int_{-\infty}^{\infty} d z_{1} \int d \vec{r}_{12} r^{2}\left(1-3 s^{2}\right) \rho_{0}^{\prime}\left(z_{1}\right) \rho_{1}\left(z_{2}\right) \\
& \times C_{0}\left(z_{1}, z_{2}, r\right) \\
& +\frac{k_{\mathrm{B}} T}{48} \int_{-\infty}^{\infty} d z_{1} \int d \vec{r}_{12} r^{4}\left(1-s^{4}\right) \rho_{0}^{\prime}\left(z_{1}\right) \rho_{0}^{\prime}\left(z_{2}\right) \\
& \times C_{0}\left(z_{1}, z_{2}, r\right) .
\end{aligned}
$$

The analysis for the $1 / R^{4}$ term in Eq. (26) for the cylindrical interface leads to Eq. (C9) in Appendix C. This gives the following two expressions for the bending rigidity $k$ :

$$
\begin{aligned}
k= & -\frac{k_{\mathrm{B}} T}{3} \int_{-\infty}^{\infty} d z_{1} \int d \vec{r}_{12} r^{2}\left(1-s^{2}\right) \sin ^{2} \varphi\left[\rho_{c}^{\prime}\left(z_{1}\right) \rho_{c}^{\prime}\left(z_{2}\right)\right. \\
& \left.\times C_{c}\left(z_{1}, z_{2}, \varphi, r\right)\right]_{2} \\
& -\frac{4 k_{\mathrm{B}} T}{3} \int_{-\infty}^{\infty} d z_{1} \int d \vec{r}_{12} r s \rho_{0}^{\prime}\left(z_{1}\right) \rho_{c, 2}\left(z_{2}\right) C_{0}\left(z_{1}, z_{2}, r\right) \\
& -\frac{k_{\mathrm{B}} T}{6} \int_{-\infty}^{\infty} d z_{1} \int d \vec{r}_{12} r s \rho_{1}\left(z_{2}\right)\left[\rho_{s}^{\prime}\left(z_{1}\right) C_{s}\left(z_{1}, z_{2}, r\right)\right]_{1} \\
& -\frac{k_{\mathrm{B}} T}{4} \int_{-\infty}^{\infty} d z_{1} \int d \vec{r}_{12} r^{2}\left(1-s^{2}\right) z_{1}^{2} \rho_{0}^{\prime}\left(z_{1}\right) \rho_{0}^{\prime}\left(z_{2}\right) \\
& \times C_{0}\left(z_{1}, z_{2}, r\right) \\
& +\frac{k_{\mathrm{B}} T}{64} \int_{-\infty}^{\infty} d z_{1} \int d \vec{r}_{12} r^{4}\left(1-s^{2}\right)\left(1+3 s^{2}\right) \rho_{0}^{\prime}\left(z_{1}\right) \rho_{0}^{\prime}\left(z_{2}\right) \\
& \times C_{0}\left(z_{1}, z_{2}, r\right)
\end{aligned}
$$

and

$$
\begin{aligned}
k= & -\frac{k_{\mathrm{B}} T}{12} \int_{-\infty}^{\infty} d z_{1} \int d \vec{r}_{12} r^{2}\left(1-s^{2}\right) z_{1}\left[\rho_{s}^{\prime}\left(z_{1}\right) \rho_{s}^{\prime}\left(z_{2}\right)\right. \\
& \left.\times C_{s}\left(z_{1}, z_{2}, r\right)\right]_{1}
\end{aligned}
$$




$$
\begin{aligned}
& -\frac{k_{\mathrm{B}} T}{6} \int_{-\infty}^{\infty} d z_{1} \int d \vec{r}_{12} r s z_{1} \rho_{0}^{\prime}\left(z_{1}\right) \rho_{1}\left(z_{2}\right) C_{0}\left(z_{1}, z_{2}, r\right) \\
& +\frac{k_{\mathrm{B}} T}{24} \int_{-\infty}^{\infty} d z_{1} \int d \vec{r}_{12} r^{2}\left(1-3 s^{2}\right) \rho_{0}^{\prime}\left(z_{1}\right) \rho_{1}\left(z_{2}\right) \\
& \times C_{0}\left(z_{1}, z_{2}, r\right) \\
& +\frac{k_{\mathrm{B}} T}{8} \int_{-\infty}^{\infty} d z_{1} \int d \vec{r}_{12} r^{2}\left(1-s^{2}\right) z_{1}^{2} \rho_{0}^{\prime}\left(z_{1}\right) \rho_{0}^{\prime}\left(z_{2}\right) \\
& \times C_{0}\left(z_{1}, z_{2}, r\right) \\
& +\frac{k_{\mathrm{B}} T}{64} \int_{-\infty}^{\infty} d z_{1} \int d \vec{r}_{12} r^{4}\left(1-s^{2}\right)^{2} \rho_{0}^{\prime}\left(z_{1}\right) \rho_{0}^{\prime}\left(z_{2}\right) \\
& \times C_{0}\left(z_{1}, z_{2}, r\right) .
\end{aligned}
$$

Combining Eqs. (34) and (36) then leads to the following expression for $\bar{k}$ :

$$
\begin{aligned}
\bar{k}= & -\frac{k_{\mathrm{B}} T}{4} \int_{-\infty}^{\infty} d z_{1} \int d \vec{r}_{12} r^{2}\left(1-s^{2}\right) z_{1}^{2} \rho_{0}^{\prime}\left(z_{1}\right) \rho_{0}^{\prime}\left(z_{2}\right) \\
& \times C_{0}\left(z_{1}, z_{2}, r\right) \\
& -\frac{k_{\mathrm{B}} T}{96} \int_{-\infty}^{\infty} d z_{1} \int d \vec{r}_{12} r^{4}\left(1-s^{2}\right)\left(1-5 s^{2}\right) \rho_{0}^{\prime}\left(z_{1}\right) \rho_{0}^{\prime}\left(z_{2}\right) \\
& \times C_{0}\left(z_{1}, z_{2}, r\right) .
\end{aligned}
$$

The expression for the Tolman length in Eq. (32) and the expressions for the rigidity constants in Eqs. (36) and (37) are the final results of this article.

\section{DISCUSSION}

For the surface tension of the planar interface, one may distinguish three types of microscopic expressions:

- Mean-field or DFT expressions of which the van der Waals squared-gradient expression is an example. These are approximate in nature but are widely used since they are relatively easy to evaluate (numerically).

- The Kirkwood-Buff virial expression, which is exact and widely used to determine the surface tension in computer simulations.

- The Triezenberg-Zwanzig expression in terms of the direct correlation function. Although the evaluation of the surface tension using this expression requires access to the direct correlation function, it has the advantage that it is an exact expression and the short-ranged nature of the direct correlation function has proven to be helpful to settle fundamental issues such as the influence of capillary waves on the structure and tension of a planar interface in the limit of vanishing gravitational field. ${ }^{30-32}$
There exists a great level of consistency between these three types of expressions for the surface tension. Although it is quite elaborate to show the equivalence of the TriezenbergZwanzig and Kirkwood-Buff expression for the surface tension, ${ }^{33}$ it is quite more simple to show how they are both consistent with mean-field expressions.

Ever since the introduction of the coefficients $\delta, k$, and $\bar{k}$ that appear in the curvature expansion of the surface tension by Tolman ${ }^{13}$ and then later by Helfrich, ${ }^{14}$ it has been the goal to formulate the same type of microscopic expressions and to achieve the same level of consistency as it exists for the surface tension of the planar interface. The road to the realization of this goal started with the formulation of squared-gradient expressions, first for the Tolman length by Fisher and Wortis in $1984^{34}$ and then for the rigidity constants by Gompper and Zschocke ${ }^{35}$ and by us ${ }^{16}$ in the early 1990 s. These squared-gradient expressions were then shown to be embedded in more general DFT expressions in $1998 .^{17,24} \mathrm{By}$ that time, also the virial expressions for $\delta, k$, and $\bar{k}$, analogous to the Kirkwood-Buff formula for the surface tension, were formulated ${ }^{15}$ and it was verified that they reduce to the DFT expressions when a mean-field approximation for the pair density is made.

Important progress was also made in the formulation of Triezenberg-Zwanzig-like expressions in terms of the direct correlation function. Even before the work by Fisher and Wortis in 1984, Henderson and co-workers ${ }^{18-20}$ derived an expression for $\sigma(R)$ correct to first order in the expansion in $1 / R$. Although it was not shown explicitly at the time, the expression for the Tolman length in Eq. (29) can be extracted from their analysis, even though the reduction to the more simple expression in Eq. (32) could not yet be made. Further progress was then made by Parry and Boulter, ${ }^{25}$ who extended the original analysis by Triezenberg-Zwanzig and derived the analogous expression for the bending rigidity in Eq. (10). To understand the status of this expression, it is important to realize that the very nature of the rigidity constants $k$ and $\bar{k}$ as the change in free energy due to the curvature of the interface, immediately leads to the following two rather subtle issues:

- The curvature of the surface, as described by the total and Gaussian curvatures $J$ and $K$, is not uniquely defined simply because the allocation of the position of the dividing surface cannot be made unambiguously. Always, some procedure to locate the dividing surface either by an integral constraint (equimolar surface) or by a crossing constraint has to be chosen. For the surface tension (and Tolman length), this observation bears no consequences but the rigidity constants are intrinsically linked to some choice for the location of the dividing surface. For example, the expressions for $k$ and $\bar{k}$ in Eqs. (36) and (37) are derived using the equimolar surface as dividing surface (Eq. (A12)).

- The system's response to curvature, and therefore the value of the rigidity constants, depends on the way this curvature is induced. Most straightforwardly it is induced by uniformly changing the value of the chemical potential (equilibrium route) - the mean-field and virial expressions are all derived in this way - but one 
could also envision the application of a non-uniform external field (fluctuation route). The corresponding values of the rigidity constants then differ as demonstrated previously in the context of DFT. ${ }^{22,36}$ The implication is that the equivalence of the equilibrium route and the fluctuation route, as it exists for the derivation of the Triezenberg-Zwanzig expression for the surface tension of the planar interface, does not exist for the derivation of Triezenberg-Zwanzig-like expression for the rigidity constants.

The expression for the bending rigidity by Parry and Boulter ${ }^{25}$ is derived in the fluctuation route, which leaves the derivation of Triezenberg-Zwanzig-like expressions for the Tolman length $\delta$ and the rigidity constants $k$ and $\bar{k}$ in the equilibrium route as the final piece of the puzzle. In this article, the expressions sought after are finally derived by extending the analysis by Henderson and co-workers. ${ }^{18-20}$ For the Tolman length, our final result is the expression in Eq. (32). For the rigidity constants our final expressions are presented in Eqs. (36) and (37).

The Triezenberg-Zwanzig-like expressions for the Tolman length and rigidity constants possess, like the Triezenberg-Zwanzig expression for the surface tension of the planar interface itself, certain advantages with respect to other approaches. Foremost, the TZ-like expressions are exact expressions which means that they are valid beyond the meanfield approximation. This is not merely a quantitative issue since it is well-known that the presence of capillary waves is not fully incorporated in mean-field theory as it fails, for instance, to capture the divergence of the interfacial width in the limit of vanishing gravitational field. ${ }^{4,30}$ A further advantage of the TZ-like expressions is that the direct correlation function featured is short-ranged even in the situation that the capillary length diverges and the range of the pair density correlation function becomes infinite. This is instrumental in addressing the influence of capillary waves on the surface tension of non-planar interfaces. For the surface tension of the planar interface it is well-established that capillary waves reduce the mean-field value for the surface tension by as much as $20 \%$, but the same analysis for the Tolman length or the rigidity constants has not been carried out thus far.

\section{ACKNOWLEDGMENTS}

This article is dedicated to the occasion of the retirement of Jim Henderson, whose scientific heritage has been and still is a source of insight and inspiration to me.

\section{APPENDIX A: DENSITY FUNCTIONAL THEORY EXPRESSIONS}

In this appendix, we present the results of density functional theory for $\delta, k$, and $\bar{k}$ (details of the analysis can be found in Ref. 24) and show how the Triezenberg-Zwanziglike expressions in terms of the direct correlation function reduce to the DFT expressions when a mean-field approximation is made.
The expression for the (grand) free energy in DFT is based on the division into a hard-sphere reference system plus attractive forces described by an interaction potential $U_{\text {att }}(r)$. It is the following functional of the density: ${ }^{3-6}$

$$
\begin{aligned}
\Omega[\rho]= & \int d \vec{r}\left[f_{\mathrm{hs}}(\rho)-\mu \rho(\vec{r})\right] \\
& +\frac{1}{2} \int d \vec{r}_{1} \int d \vec{r}_{12} U_{\mathrm{att}}(r) \rho\left(\vec{r}_{1}\right) \rho\left(\vec{r}_{2}\right),
\end{aligned}
$$

where $f_{\text {hs }}(\rho)$ is the free energy density of the hard-sphere reference system with uniform density $\rho$. This functional is based on a local density approximation for the hard-sphere reference fluid, but more sophisticated approaches exist using weighted-density ${ }^{6}$ or fundamental measure theory. ${ }^{37}$

The Euler-Lagrange equation that minimizes the above free energy is given by

$$
f_{\mathrm{hs}}^{\prime}(\rho)=\mu-\int d \vec{r}_{12} U_{\text {att }}(r) \rho\left(\vec{r}_{2}\right) .
$$

To derive expressions for the curvature coefficients $\delta, k$, and $\bar{k}$, one needs to consider the expansion of the density and free energy in Eq. (A1) to second order in $1 / R$ for spherically and cylindrically shaped liquid droplets. For instance, the expansion of the density profile of the spherical droplet reads

$$
\rho_{s}(r)=\rho_{0}(z)+\frac{1}{R} \rho_{s, 1}(z)+\frac{1}{R^{2}} \rho_{s, 2}(z)+\ldots
$$

The leading order correction to the density profile of the spherical interface is twice that of the cylindrical interface, so it is convenient to define $\rho_{1}(z) \equiv \rho_{s, 1}(z)=2 \rho_{c, 1}(z)$, where $z \equiv r-R$.

The coefficients in the curvature expansion of the density are determined from the curvature expansion of the EulerLagrange equation in Eq. (A2). The result is that the (planar) density profile $\rho_{0}(z)$ is determined from solving:

$$
\mu_{\mathrm{coex}}=f_{\mathrm{hs}}^{\prime}\left(\rho_{0}\right)+\int d \vec{r}_{12} U_{\mathrm{att}}(r) \rho_{0}\left(z_{2}\right)
$$

and $\rho_{1}(z)$ follows from solving:

$$
\begin{aligned}
\mu_{1}= & f_{\mathrm{hs}}^{\prime \prime}\left(\rho_{0}\right) \rho_{1}\left(z_{1}\right)+\int d \vec{r}_{12} U_{\mathrm{att}}(r) \\
& \times\left[\rho_{1}\left(z_{2}\right)+\frac{r^{2}}{2}\left(1-s^{2}\right) \rho_{0}^{\prime}\left(z_{2}\right)\right],
\end{aligned}
$$

where $\mu_{1}=2 \sigma / \Delta \rho .^{16,36}$ For the evaluation of the curvature coefficients it turns out to be sufficient to determine the density profiles $\rho_{0}(z)$ and $\rho_{1}(z)$ only.

Expressions for the curvature coefficients are now derived from the expansion of the free energy in Eq. (A1) to second order in $1 / R$ and by comparing the result to the expansions in Eq. (6). This leads to the following two equivalent 
expressions for the Tolman length: ${ }^{17}$

$$
\delta \sigma=\frac{1}{4} \int_{-\infty}^{\infty} d z_{1} \int d \vec{r}_{12} U_{\text {att }}(r) r^{2}\left(1-s^{2}\right) \rho_{0}^{\prime}\left(z_{1}\right) \rho_{1}^{\prime}\left(z_{2}\right)
$$

and

$$
\begin{aligned}
\delta \sigma= & \frac{1}{4} \int_{-\infty}^{\infty} d z_{1} \int d \vec{r}_{12} U_{\mathrm{att}}(r) r^{2}\left(1-s^{2}\right) z_{1} \rho_{0}^{\prime}\left(z_{1}\right) \rho_{0}^{\prime}\left(z_{2}\right) \\
& -\frac{\mu_{1}}{2} \int_{-\infty}^{\infty} d z z \rho_{0}^{\prime}(z)
\end{aligned}
$$

The second order term in the expansion of the free energy for the spherical interface, leads to the following expression for the combination of the rigidity constants, $2 k+\bar{k}:^{24}$

$$
\begin{aligned}
2 k+\bar{k}= & \frac{1}{4} \int_{-\infty}^{\infty} d z_{1} \int d \vec{r}_{12} U_{\mathrm{att}}(r) r^{2}\left(1-s^{2}\right) \rho_{0}^{\prime}\left(z_{1}\right) \rho_{1}\left(z_{2}\right) \\
& -\frac{1}{4} \int_{-\infty}^{\infty} d z_{1} \int d \vec{r}_{12} U_{\mathrm{att}}(r) r^{2}\left(1-s^{2}\right) z_{1}^{2} \rho_{0}^{\prime}\left(z_{1}\right) \rho_{0}^{\prime}\left(z_{2}\right) \\
& +\frac{1}{48} \int_{-\infty}^{\infty} d z_{1} \int d \vec{r}_{12} U_{\mathrm{att}}(r) r^{4}\left(1-s^{4}\right) \rho_{0}^{\prime}\left(z_{1}\right) \rho_{0}^{\prime}\left(z_{2}\right) \\
& +\int_{-\infty}^{\infty} d z\left[\frac{\mu_{1}}{2} z \rho_{1}^{\prime}(z)+\mu_{1} z^{2} \rho_{0}^{\prime}(z)+\mu_{s, 2} z \rho_{0}^{\prime}(z)\right]
\end{aligned}
$$

where $\mu_{s, 2}=-\sigma \Delta \rho_{1} /(\Delta \rho)^{2}-2 \delta \sigma / \Delta \rho^{16,36}$ with $\Delta \rho_{1}$ $\equiv \rho_{1, \ell}-\rho_{1, v}$. An alternative expression that contains no reference to the chemical potential is also derived in Ref. 24:

$$
\begin{aligned}
2 k+\bar{k}= & -\frac{1}{2} \int_{-\infty}^{\infty} d z_{1} \int d \vec{r}_{12} U_{\text {att }}(r) r^{2}\left(1-s^{2}\right) \rho_{0}^{\prime}\left(z_{1}\right) \rho_{s, 2}^{\prime}\left(z_{2}\right) \\
& -\frac{1}{4} \int_{-\infty}^{\infty} d z_{1} \int d \vec{r}_{12} U_{\text {att }}(r) r^{2}\left(1-s^{2}\right) \rho_{1}^{\prime}\left(z_{1}\right) \rho_{1}^{\prime}\left(z_{2}\right) \\
& -\frac{1}{4} \int_{-\infty}^{\infty} d z_{1} \int d \vec{r}_{12} U_{\text {att }}(r) r^{2}\left(1-s^{2}\right) z_{1}^{2} \rho_{0}^{\prime}\left(z_{1}\right) \rho_{0}^{\prime}\left(z_{2}\right) \\
& +\frac{1}{48} \int_{-\infty}^{\infty} d z_{1} \int d \vec{r}_{12} U_{\text {att }}(r) r^{4}\left(1-s^{4}\right) \rho_{0}^{\prime}\left(z_{1}\right) \rho_{0}^{\prime}\left(z_{2}\right) .
\end{aligned}
$$

To derive an expression for the bending rigidity $k$, an expansion of the free energy to second order in $1 / R$ is made for the cylindrical interface. For the Tolman length the expressions in Eqs. (A6) and (A7) are recovered and one finds for the bending rigidity: ${ }^{24}$

$$
\begin{aligned}
k= & \frac{1}{8} \int_{-\infty}^{\infty} d z_{1} \int d \vec{r}_{12} U_{\text {att }}(r) r^{2}\left(1-s^{2}\right) \rho_{0}^{\prime}\left(z_{1}\right) \rho_{1}\left(z_{2}\right) \\
& +\frac{1}{64} \int_{-\infty}^{\infty} d z_{1} \int d \vec{r}_{12} U_{\text {att }}(r) r^{4}\left(1-s^{2}\right)^{2} \rho_{0}^{\prime}\left(z_{1}\right) \rho_{0}^{\prime}\left(z_{2}\right) \\
& +\int_{-\infty}^{\infty} d z\left[\frac{\mu_{1}}{4} z \rho_{1}^{\prime}(z)+\frac{\mu_{1}}{2} z^{2} \rho_{0}^{\prime}(z)+2 \mu_{c, 2} z \rho_{0}^{\prime}(z)\right],
\end{aligned}
$$

where $\mu_{c, 2}=-\sigma \Delta \rho_{1} /(2 \Delta \rho)^{2} \cdot{ }^{16,36}$ As an alternative expression for $k$ that contains no reference to the chemical potential is $^{24}$

$$
\begin{aligned}
k= & -\int_{-\infty}^{\infty} d z_{1} \int d \vec{r}_{12} U_{\text {att }}(r) r^{2}\left(1-s^{2}\right) \rho_{0}^{\prime}\left(z_{1}\right) \rho_{c, 2}^{\prime}\left(z_{2}\right) \\
& -\frac{1}{8} \int_{-\infty}^{\infty} d z_{1} \int d \vec{r}_{12} U_{\text {att }}(r) r^{2}\left(1-s^{2}\right) \rho_{1}^{\prime}\left(z_{1}\right) \rho_{1}^{\prime}\left(z_{2}\right) \\
& -\frac{1}{4} \int_{-\infty}^{\infty} d z_{1} \int d \vec{r}_{12} U_{\text {att }}(r) r^{2}\left(1-s^{2}\right)^{2} z_{1}^{2} \rho_{0}^{\prime}\left(z_{1}\right) \rho_{0}^{\prime}\left(z_{2}\right) \\
& +\frac{1}{64} \int_{-\infty}^{\infty} d z_{1} \int d \vec{r}_{12} U_{\text {att }}(r) r^{4}\left(1-s^{2}\right)\left(1+3 s^{2}\right) \rho_{0}^{\prime}\left(z_{1}\right) \rho_{0}^{\prime}\left(z_{2}\right)
\end{aligned}
$$

These expressions are derived in Ref. 24 without reference to any particular choice for the location of the dividing surface. In the present analysis, it is important that the radius $R$ is defined according to the equimolar radius in Eq. (19). Expansion of Eq. (19) to first order in $1 / R$ then leads to the following two conditions for the expanded profiles $\rho_{0}(z)$ and $\rho_{1}(z):^{16}$

$$
\int_{-\infty}^{\infty} d z z \rho_{0}^{\prime}(z)=0 \text { and } \int_{-\infty}^{\infty} d z z \rho_{1}^{\prime}(z)=-\int_{-\infty}^{\infty} d z z^{2} \rho_{0}^{\prime}(z) .
$$

By making explicit use of this property for $\rho_{0}(z)$, Eq. (A7) may be rewritten as

$$
\delta \sigma=\frac{1}{4} \int_{-\infty}^{\infty} d z_{1} \int d \vec{r}_{12} U_{\text {att }}(r) r^{2}\left(1-s^{2}\right) z_{1} \rho_{0}^{\prime}\left(z_{1}\right) \rho_{0}^{\prime}\left(z_{2}\right) .
$$

Furthermore, using the properties for $\rho_{0}(z)$ and $\rho_{1}(z)$ in Eq. (A12), the expression for $2 k+\bar{k}$ in Eq. (A8) is rewritten as

$$
\begin{aligned}
2 k+\bar{k}= & -\frac{1}{4} \int_{-\infty}^{\infty} d z_{1} \int d \vec{r}_{12} U_{\text {att }}(r) r^{2}\left(1-s^{2}\right) \\
& \times\left(z_{1}+z_{2}\right) \rho_{0}^{\prime}\left(z_{1}\right) \rho_{1}^{\prime}\left(z_{2}\right) \\
& +\frac{1}{48} \int_{-\infty}^{\infty} d z_{1} \int d \vec{r}_{12} U_{\text {att }}(r) r^{4}\left(1-s^{4}\right) \rho_{0}^{\prime}\left(z_{1}\right) \rho_{0}^{\prime}\left(z_{2}\right),
\end{aligned}
$$


while Eq. (A10) is rewritten as

$$
\begin{aligned}
k= & -\frac{1}{8} \int_{-\infty}^{\infty} d z_{1} \int d \vec{r}_{12} U_{\text {att }}(r) r^{2}\left(1-s^{2}\right)\left(z_{1}+z_{2}\right) \rho_{0}^{\prime}\left(z_{1}\right) \rho_{1}^{\prime}\left(z_{2}\right) \\
& +\frac{1}{8} \int_{-\infty}^{\infty} d z_{1} \int d \vec{r}_{12} U_{\text {att }}(r) r^{2}\left(1-s^{2}\right) z_{1}^{2} \rho_{0}^{\prime}\left(z_{1}\right) \rho_{0}^{\prime}\left(z_{2}\right) \\
& +\frac{1}{64} \int_{-\infty}^{\infty} d z_{1} \int d \vec{r}_{12} U_{\text {att }}(r) r^{4}\left(1-s^{2}\right)^{2} \rho_{0}^{\prime}\left(z_{1}\right) \rho_{0}^{\prime}\left(z_{2}\right) .
\end{aligned}
$$

Next, we verify that the expressions for $\delta, k$, and $2 k+\bar{k}$ as presented in Sec. IV reduce to the DFT expressions listed above. To show the equivalence of the Triezenberg-Zwanzig and DFT expression for the surface tension of the planar interface, one approximates the direct correlation function by the attractive part of the interaction potential: ${ }^{4,5}$

$$
k_{\mathrm{B}} T C_{0}\left(z_{1}, z_{2}, r\right) \rightarrow f_{\mathrm{hs}}^{\prime \prime}\left(\rho_{0}\right) \delta\left(\vec{r}_{2}-\vec{r}_{1}\right)+U_{\mathrm{att}}(r) .
$$

Inserting this approximation into the $\mathrm{TZ}$ expression for the surface tension in Eq. (5) then immediately leads to the DFT expression in Eq. (2).

Equation (A16) is easily generalized to non-planar geometries by replacing $C_{0}$ and $\rho_{0}$ by the corresponding nonplanar direct correlation function and density profile. Since all the curvature expressions feature a factor $r^{2}$ times the direct correlation, we may disregard the delta-function contribution in Eq. (A16). Using the fact that the interaction potential is independent of $R$, we have the following replacements:

$$
\begin{aligned}
k_{\mathrm{B}} T\left[\rho_{s}^{\prime} C_{s}\right]_{1} \rightarrow & \rho_{1}^{\prime}\left(z_{1}\right) U_{\mathrm{att}}(r), \\
k_{\mathrm{B}} T\left[\rho_{s}^{\prime} \rho_{s}^{\prime} C_{s}\right]_{1} \rightarrow & {\left[\rho_{0}^{\prime}\left(z_{1}\right) \rho_{1}^{\prime}\left(z_{2}\right)+\rho_{1}^{\prime}\left(z_{1}\right) \rho_{0}^{\prime}\left(z_{2}\right)\right] U_{\mathrm{att}}(r), } \\
k_{\mathrm{B}} T\left[\rho_{s}^{\prime} \rho_{s}^{\prime} C_{s}\right]_{2} \rightarrow & {\left[\rho_{0}^{\prime}\left(z_{1}\right) \rho_{s, 2}^{\prime}\left(z_{2}\right)+\rho_{1}^{\prime}\left(z_{1}\right) \rho_{1}^{\prime}\left(z_{2}\right)\right.} \\
& \left.+\rho_{s, 2}^{\prime}\left(z_{1}\right) \rho_{0}^{\prime}\left(z_{2}\right)\right] U_{\mathrm{att}}(r), \\
k_{\mathrm{B}} T\left[\rho_{c}^{\prime} \rho_{c}^{\prime} C_{c}\right]_{2} \rightarrow & {\left[\rho_{0}^{\prime}\left(z_{1}\right) \rho_{c, 2}^{\prime}\left(z_{2}\right)+\rho_{1}^{\prime}\left(z_{1}\right) \rho_{1}^{\prime}\left(z_{2}\right) / 4\right.} \\
& \left.+\rho_{c, 2}^{\prime}\left(z_{1}\right) \rho_{0}^{\prime}\left(z_{2}\right)\right] U_{\mathrm{att}}(r) .
\end{aligned}
$$

Another property that is helpful in verifying that the expressions in Sec. IV reduce to the DFT expressions, is integration by parts with respect to the parameter $s$. It is easily verified that

$$
\begin{aligned}
\int_{-1}^{1} d s r s \rho_{\mathrm{x}}\left(z_{2}\right) & =\int_{-1}^{1} d s \frac{r^{2}}{2}\left(1-s^{2}\right) \rho_{\mathrm{x}}^{\prime}\left(z_{2}\right), \\
\int_{-1}^{1} d s\left(1-3 s^{2}\right) \rho_{\mathrm{x}}\left(z_{2}\right) & =-\int_{-1}^{1} d s r s\left(1-s^{2}\right) \rho_{\mathrm{x}}^{\prime}\left(z_{2}\right) .
\end{aligned}
$$

With the help of Eqs. (A17) and (A18), we may now show that: (i) For the Tolman length $\delta$ : Eq. (29) reduces to Eq. (A6) and Eq. (32) reduces to Eq. (A13); (ii) for the combination $2 k+\bar{k}$ : Eq. (33) reduces to Eq. (A9) and Eq. (34) reduces to Eq. (A14); (iii) for the bending rigidity $k$ : Eq. (35) reduces to Eq. (A11) and Eq. (36) reduces to Eq. (A15).

\section{APPENDIX B: EQUILIBRIUM ROUTE-SPHERICAL DROPLET}

In this appendix, we insert the expression for $\delta \rho_{s}(r)$ in Eq. (28) into Eq. (25) and expand to order $\mathcal{O}\left(1 / R^{4}\right)$. Three terms result that we shall investigate separately:

$$
\begin{aligned}
\frac{2 \sigma}{R^{2}}-\frac{4 \delta \sigma}{R^{3}}= & -k_{\mathrm{B}} T \int_{0}^{\infty} d r_{1} \int d \vec{r}_{2} C_{s}\left(r_{1}, r_{2}, r\right) \rho_{s}^{\prime}\left(r_{1}\right) \rho_{s}^{\prime}\left(r_{2}\right) \\
& -\frac{k_{\mathrm{B}} T}{R^{2}} \int_{0}^{\infty} d r_{1} \int d \vec{r}_{2} C_{s}\left(r_{1}, r_{2}, r\right) \rho_{s}^{\prime}\left(r_{1}\right) \rho_{1}\left(r_{2}\right) \\
& -\frac{2 k_{\mathrm{B}} T}{R^{3}} \int_{0}^{\infty} d r_{1} \int d \vec{r}_{2} C_{s}\left(r_{1}, r_{2}, r\right) \rho_{s}^{\prime}\left(r_{1}\right) \rho_{s, 2}\left(r_{2}\right) .
\end{aligned}
$$

The second Yvon-equation in Eq. (14) in spherical symmetry reads

$$
V_{\text {ext }}^{\prime}\left(r_{1}\right) \hat{r}_{1}=\int d \vec{r}_{2} C_{s}\left(r_{1}, r_{2}, r\right) \rho_{s}^{\prime}\left(r_{2}\right) \hat{r}_{2} .
$$

Considering the component in the $\hat{r}_{1}$ direction and considering the limit of a vanishing external field, we have that

$$
0=\int d \vec{r}_{2} C_{s}\left(r_{1}, r_{2}, r\right) \rho_{s}^{\prime}\left(r_{2}\right) \frac{\left(r_{1}+s r\right)}{r_{2}},
$$

where we have used that $\hat{r}_{1} \cdot \hat{r}_{2}=\left(r_{1}+s r\right) / r_{2}$. This is conveniently rewritten as

$$
\begin{aligned}
& \int d \vec{r}_{2} C_{s}\left(r_{1}, r_{2}, r\right) \rho_{s}^{\prime}\left(r_{2}\right) \\
& \quad=\int d \vec{r}_{2} C_{s}\left(r_{1}, r_{2}, r\right) \rho_{s}^{\prime}\left(r_{2}\right)\left[1-\frac{\left(r_{1}+s r\right)}{r_{2}}\right] .
\end{aligned}
$$

The term in square brackets is expanded in the inverse radius to give

$$
\begin{aligned}
1-\frac{\left(r_{1}+s r\right)}{r_{2}}= & \frac{r^{2}}{2 R^{2}}\left(1-s^{2}\right) \\
& \times\left[1-\frac{2}{R}\left(z_{1}+s r\right)+\frac{3}{R^{2}}\left(z_{1}^{2}+2 z_{1} s r\right.\right. \\
& \left.\left.\times-\frac{r^{2}}{4}\left(1-5 s^{2}\right)\right)+\ldots\right]
\end{aligned}
$$

where it is reminded that $z_{1} \equiv r_{1}-R$. For the second and third terms in Eq. (B1), we can use that

$$
\begin{aligned}
& \int_{0}^{\infty} d r_{1} \int d \vec{r}_{2} C_{s}\left(r_{1}, r_{2}, r\right) \rho_{s}^{\prime}\left(r_{1}\right) \rho_{\mathrm{x}}\left(r_{2}\right) \\
& \quad=\int_{0}^{\infty} d r_{1} \int d \vec{r}_{2} C_{s}\left(r_{1}, r_{2}, r\right) \rho_{s}^{\prime}\left(r_{2}\right) \rho_{\mathrm{x}}\left(r_{1}\right)\left[\frac{r_{1}^{2}}{r_{2}^{2}}-\frac{\left(r_{1}+s r\right)}{r_{2}}\right] .
\end{aligned}
$$

The first term in the square brackets results after making use of the $1 \leftrightarrow 2$ symmetry of the direct correlation function. The second term in the square brackets can be added since Eq. (B4) indicates that it is equal to zero. This addition is convenient since the sum of these two terms is now of 
$\operatorname{order} \mathcal{O}(1 / R)$ :

$$
\frac{r_{1}^{2}}{r_{2}^{2}}-\frac{\left(r_{1}+s r\right)}{r_{2}}=-\frac{2 s r}{R}+\frac{2 z_{1} s r}{R^{2}}-\frac{r^{2}}{2 R^{2}}\left(1-7 s^{2}\right)+\ldots
$$

An important subtlety in the expansion of the direct correlation is the fact that the second argument in $C_{s}\left(r_{1}, r_{2}, r\right)$ is expanded around $r_{2}=r_{1}+r s$. The way this is done is explained in Ref. 15 where the same mathematical technique is used for the pair density. The result is that

$$
\begin{aligned}
C_{s}\left(r_{1}, r_{2}, r\right)= & {\left[1+\frac{r^{2}\left(1-s^{2}\right)}{2 R} \frac{d}{r d s}+\frac{r^{4}\left(1-s^{2}\right)^{2}}{8 R^{2}} \frac{d^{2}}{r^{2} d s^{2}}\right.} \\
& \left.-\frac{r^{2}\left(1-s^{2}\right) z_{2}}{2 R^{2}} \frac{d}{r d s}+\ldots\right] C_{s}\left(r_{1}, r_{1}+r s, r\right) .
\end{aligned}
$$

A systematic expansion in $1 / R$ of the right-hand-side in Eq. (B1) can now be made. After some algebra (using Eq. (D4) in Appendix D) and by comparing with the lefthand-side of Eq. (B1), we obtain the TZ expression for $\sigma$ in Eq. (5) and find for the Tolman length the following expression:

$$
\delta \sigma=\frac{k_{\mathrm{B}} T}{8} \int_{-\infty}^{\infty} d z_{1} \int d \vec{r}_{12} r^{2}\left(1-s^{2}\right)\left[\rho_{s}^{\prime}\left(z_{1}\right) \rho_{s}^{\prime}\left(z_{2}\right) C_{s}\left(z_{1}, z_{2}, r\right)\right]_{1} .
$$

From the $1 / R^{4}$ term in the expansion of the right-hand-side of Eq. (B1), we have

$$
\left.\begin{array}{rl}
0= & -\frac{k_{\mathrm{B}} T}{12} \int_{-\infty}^{\infty} d z_{1} \int d \vec{r}_{12} r^{2}\left(1-s^{2}\right)\left[\rho_{s}^{\prime}\left(z_{1}\right) \rho_{s}^{\prime}\left(z_{2}\right) C_{s}\left(z_{1}, z_{2}, r\right)\right]_{2} \\
& -\frac{2 k_{\mathrm{B}} T}{3} \int_{-\infty}^{\infty} d z_{1} \int d \vec{r}_{12} r s \rho_{0}^{\prime}\left(z_{1}\right) \rho_{s, 2}\left(z_{2}\right) C_{0}\left(z_{1}, z_{2}, r\right) \\
& -\frac{k_{\mathrm{B}} T}{3} \int_{-\infty}^{\infty} d z_{1} \int d \vec{r}_{12} r s \rho_{1}\left(z_{2}\right)\left[\rho_{s}^{\prime}\left(z_{1}\right) C_{s}\left(z_{1}, z_{2}, r\right)\right]_{1} \\
& -\frac{k_{\mathrm{B}} T}{4} \int_{-\infty}^{\infty} d z_{1} \int d \vec{r}_{12} r^{2}\left(1-s^{2}\right) z_{1}^{2} \rho_{0}^{\prime}\left(z_{1}\right) \rho_{0}^{\prime}\left(z_{2}\right) C_{0}\left(z_{1}, z_{2}, r\right) \\
& +\frac{k_{\mathrm{B}} T}{48} \int_{-\infty}^{\infty} d z_{1} \int d \vec{r}_{12} r^{4}\left(1-s^{4}\right) \rho_{0}^{\prime}\left(z_{1}\right) \rho_{0}^{\prime}\left(z_{2}\right) C_{0}\left(z_{1}, z_{2}, r\right) \\
& +\frac{k_{\mathrm{B}} T}{6} \int_{-\infty}^{\infty} d z_{1} \int d \vec{r}_{12} r\left(1-s^{2}\right) z_{1}\left[\rho_{s}^{\prime}\left(z_{1}\right) \rho_{s}^{\prime}\left(z_{2}\right) C_{s}\left(z_{1}, z_{2}, r\right)\right]_{1} \\
& +\frac{k_{\mathrm{B}} T}{3} \int_{-\infty}^{\infty} d z_{1} \int d \vec{r}_{12} r s z_{1} \rho_{0}^{\prime}\left(z_{1}\right) \rho_{1}\left(z_{2}\right) C_{0}\left(z_{1}, z_{2}, r\right) \\
& -\frac{k_{\mathrm{B}} T}{12} \int_{-\infty}^{\infty} d z_{1} \int d \vec{r}_{12} r^{2}\left(1-3 s^{2}\right) \rho_{0}^{\prime}\left(z_{1}\right) \rho_{1}\left(z_{2}\right) C_{0}\left(z_{1}, z_{2}, r\right) \\
& -\frac{k_{\mathrm{B}} T}{48} \int_{-\infty}^{\infty} d z_{1} \int d \vec{r}_{12} r^{4}\left(1-s^{4}\right) \rho_{0}^{\prime}\left(z_{1}\right) \rho_{0}^{\prime}\left(z_{2}\right) C_{0}\left(z_{1}, z_{2}, r\right)
\end{array}\right\}-(2 k
$$

The brackets indicate how the vanishing of this term may expressed as the sum of two separate expressions for $2 k+\bar{k}$. This division may appear rather arbitrary (the fifth and ninth terms cancel, for instance), but is constructed such that the separate contributions reduce to the DFT expressions for $2 k+\bar{k}$ as outlined in Appendix A. This means that in principle a term could be added and subtracted that has the property that it is zero when a mean-field approximation is made, such as a term that contains $C_{s, 1}\left(z_{1}, z_{2}, r\right)$ only. Since none of the terms in Eq. (B10) have this property, the presence of such a term is hard to imagine, however.

\section{APPENDIX C: EQUILIBRIUM ROUTE-CYLINDRICAL DROPLET}

In this appendix, we carry out the same analysis as in Appendix B but now for the cylindrical interface. Expanding $\rho_{c}(r)$ in $1 / R$, as in Eq. (28), gives

$$
\delta \rho_{c}(r)=-\left[\rho_{c}^{\prime}(r)+\frac{\rho_{1}(r)}{2 R^{2}}+\frac{2 \rho_{c, 2}(r)}{R^{3}}+\ldots\right] \delta R .
$$

Inserting this expression for $\delta \rho_{c}(r)$ into Eq. (26) and expanding to order $\mathcal{O}\left(1 / R^{4}\right)$, we now have

$$
\begin{aligned}
\frac{\sigma}{R^{2}} & -\frac{3 k}{2 R^{4}} \\
= & -k_{\mathrm{B}} T \int_{0}^{\infty} d r_{1} \int d \vec{r}_{2} C_{c}\left(r_{1}, r_{2}, \varphi, r\right) \rho_{c}^{\prime}\left(r_{1}\right) \rho_{c}^{\prime}\left(r_{2}\right) \\
& -\frac{k_{\mathrm{B}} T}{2 R^{2}} \int_{0}^{\infty} d r_{1} \int d \vec{r}_{2} C_{c}\left(r_{1}, r_{2}, \varphi, r\right) \rho_{c}^{\prime}\left(r_{1}\right) \rho_{1}\left(r_{2}\right) \\
& -\frac{2 k_{\mathrm{B}} T}{R^{3}} \int_{0}^{\infty} d r_{1} \int d \vec{r}_{2} C_{c}\left(r_{1}, r_{2}, \varphi, r\right) \rho_{c}^{\prime}\left(r_{1}\right) \rho_{c, 2}\left(r_{2}\right) .
\end{aligned}
$$


In the absence of an external field, the second Yvon-equation in Eq. (14) in cylindrical geometry gives that

$$
\begin{aligned}
& \int d \vec{r}_{2} C_{c}\left(r_{1}, r_{2}, \varphi, r\right) \rho_{c}^{\prime}\left(r_{2}\right) \\
& \quad=\int d \vec{r}_{2} C_{c}\left(r_{1}, r_{2}, \varphi, r\right) \rho_{c}^{\prime}\left(r_{2}\right)\left[1-\frac{\left(r_{1}+s r\right)}{r_{2}}\right] .
\end{aligned}
$$

where we have used that $\vec{\nabla}_{2} \rho_{c}\left(r_{2}\right)=\rho_{c}^{\prime}\left(r_{2}\right) \hat{r}_{2}$ and that $\hat{r}_{1} \cdot \hat{r}_{2}$ $=\left(r_{1}+s r\right) / r_{2}$. The term in square brackets can be expanded in the inverse radius to give

$$
\begin{aligned}
1-\frac{\left(r_{1}+s r\right)}{r_{2}}= & \frac{r^{2}}{2 R^{2}}\left(1-s^{2}\right) \sin ^{2} \varphi \\
& \times\left[1-\frac{2}{R}\left(z_{1}+s r\right)+\frac{3}{R^{2}}\left(z_{1}^{2}+2 z_{1} s r\right.\right. \\
& \left.\left.-\frac{r^{2}}{4}\left(1-s^{2}\right) \sin ^{2} \varphi+r^{2} s^{2}\right)+\ldots\right] .
\end{aligned}
$$

For the second and third terms in Eq. (C2), we can use that

$$
\begin{aligned}
& \int_{0}^{\infty} d r_{1} \int d \vec{r}_{2} C_{c}\left(r_{1}, r_{2}, \varphi, r\right) \rho_{c}^{\prime}\left(r_{1}\right) \rho_{\mathrm{x}}\left(r_{2}\right) \\
& \quad=\int_{0}^{\infty} d r_{1} \int d \vec{r}_{2} C_{c}\left(r_{1}, r_{2}, \varphi, r\right) \rho_{c}^{\prime}\left(r_{2}\right) \rho_{\mathrm{x}}\left(r_{1}\right)\left[\frac{r_{1}}{r_{2}}-\frac{\left(r_{1}+s r\right)}{r_{2}}\right] .
\end{aligned}
$$

The first term in the square brackets results after making use of the $1 \leftrightarrow 2$ symmetry of the direct correlation function. The second term in the square brackets can be added since Eq. (C3) indicates that it is equal to zero. This addition is convenient since the sum of these two terms is now of order $\mathcal{O}(1 / R)$ :

$$
\frac{r_{1}}{r_{2}}-\frac{\left(r_{1}+s r\right)}{r_{2}}=-\frac{s r}{R}+\frac{z_{1} s r}{R^{2}}+\frac{r^{2} s^{2}}{2 R^{2}}+\ldots
$$

Analogous to Eq. (B8), $C_{c}\left(r_{1}, r_{2}, \varphi, r\right)$ is expanded around $r_{2}$ $=r_{1}+r s$ :

$$
\begin{aligned}
C_{c}\left(r_{1}, r_{2}, \varphi, r\right) \\
=\left[1+\frac{r^{2}\left(1-s^{2}\right) \sin ^{2} \varphi}{2 R} \frac{d}{r d s}+\frac{r^{4}\left(1-s^{2}\right)^{2} \sin ^{4} \varphi}{8 R^{2}} \frac{d^{2}}{r^{2} d s^{2}}\right. \\
\\
\left.-\frac{r^{2}\left(1-s^{2}\right) z_{2} \sin ^{2} \varphi}{2 R^{2}} \frac{d}{r d s}+\ldots\right] C_{c}\left(r_{1}, r_{2}+r s, \varphi, r\right) .
\end{aligned}
$$

Again, a systematic expansion in $1 / R$ of the right-hand-side in Eq. (C2) can now be made. After some algebra and by comparing to the left-hand-side of Eq. (C2), we now obtain from the condition of the vanishing of the first order term that

$$
\begin{aligned}
0= & \left.\frac{k_{\mathrm{B}} T}{4} \int_{-\infty}^{\infty} d z_{1} \int d \vec{r}_{12} r^{2}\left(1-s^{2}\right) z_{1} \rho_{0}^{\prime}\left(z_{1}\right) \rho_{0}^{\prime}\left(z_{2}\right) C_{0}\left(z_{1}, z_{2}, r\right)\right\} \delta \sigma \\
& \left.-\frac{k_{\mathrm{B}} T}{8} \int_{-\infty}^{\infty} d z_{1} \int d \vec{r}_{12} r^{2}\left(1-s^{2}\right)\left[\rho_{s}^{\prime}\left(z_{1}\right) \rho_{s}^{\prime}\left(z_{2}\right) C_{s}\left(z_{1}, z_{2}, r\right)\right]_{1}\right\}-\delta \sigma
\end{aligned}
$$

where we have used the fact that to leading order $C_{c, 1}\left(z_{1}, z_{2}, \varphi, r\right)=\frac{1}{2} C_{s, 1}\left(z_{1}, z_{2}, r\right)$. The $1 / R^{4}$ term in Eq. (C2) now supplies us with an expression for the bending rigidity:

$$
\left.\begin{array}{rl}
k= & \frac{k_{\mathrm{B}} T}{3} \int_{-\infty}^{\infty} d z_{1} \int d \vec{r}_{12} r^{2}\left(1-s^{2}\right) \sin ^{2} \varphi\left[\rho_{c}^{\prime}\left(z_{1}\right) \rho_{c}^{\prime}\left(z_{2}\right) C_{c}\left(z_{1}, z_{2}, \varphi, r\right)\right]_{2} \\
& +\frac{4 k_{\mathrm{B}} T}{3} \int_{-\infty}^{\infty} d z_{1} \int d \vec{r}_{12} r s \rho_{0}^{\prime}\left(z_{1}\right) \rho_{c, 2}\left(z_{2}\right) C_{0}\left(z_{1}, z_{2}, r\right) \\
& +\frac{k_{\mathrm{B}} T}{6} \int_{-\infty}^{\infty} d z_{1} \int d \vec{r}_{12} r s \rho_{1}\left(z_{2}\right)\left[\rho_{s}^{\prime}\left(z_{1}\right) C_{s}\left(z_{1}, z_{2}, r\right)\right]_{1} \\
& +\frac{k_{\mathrm{B}} T}{4} \int_{-\infty}^{\infty} d z_{1} \int d \vec{r}_{12} r^{2}\left(1-s^{2}\right) z_{1}^{2} \rho_{0}^{\prime}\left(z_{1}\right) \rho_{0}^{\prime}\left(z_{2}\right) C_{0}\left(z_{1}, z_{2}, r\right) \\
& -\frac{k_{\mathrm{B}} T}{64} \int_{-\infty}^{\infty} d z_{1} \int d \vec{r}_{12} r^{4}\left(1-s^{2}\right)\left(1+3 s^{2}\right) \rho_{0}^{\prime}\left(z_{1}\right) \rho_{0}^{\prime}\left(z_{2}\right) C_{0}\left(z_{1}, z_{2}, r\right)
\end{array}\right\}-k
$$




$$
\begin{aligned}
& -\frac{k_{\mathrm{B}} T}{6} \int_{-\infty}^{\infty} d z_{1} \int d \vec{r}_{12} r^{2}\left(1-s^{2}\right) z_{1}\left[\rho_{s}^{\prime}\left(z_{1}\right) \rho_{s}^{\prime}\left(z_{2}\right) C_{s}\left(z_{1}, z_{2}, r\right)\right]_{1} \\
& -\frac{k_{\mathrm{B}} T}{3} \int_{-\infty}^{\infty} d z_{1} \int d \vec{r}_{12} r s z_{1} \rho_{0}^{\prime}\left(z_{1}\right) \rho_{1}\left(z_{2}\right) C_{0}\left(z_{1}, z_{2}, r\right) \\
& +\frac{k_{\mathrm{B}} T}{12} \int_{-\infty}^{\infty} d z_{1} \int d \vec{r}_{12} r^{2}\left(1-3 s^{2}\right) \rho_{0}^{\prime}\left(z_{1}\right) \rho_{1}\left(z_{2}\right) C_{0}\left(z_{1}, z_{2}, r\right) \\
& +\frac{k_{\mathrm{B}} T}{4} \int_{-\infty}^{\infty} d z_{1} \int d \vec{r}_{12} r^{2}\left(1-s^{2}\right) z_{1}^{2} \rho_{0}^{\prime}\left(z_{1}\right) \rho_{0}^{\prime}\left(z_{2}\right) C_{0}\left(z_{1}, z_{2}, r\right) \\
& +\frac{k_{\mathrm{B}} T}{32} \int_{-\infty}^{\infty} d z_{1} \int d \vec{r}_{12} r^{4}\left(1-s^{2}\right)^{2} \rho_{0}^{\prime}\left(z_{1}\right) \rho_{0}^{\prime}\left(z_{2}\right) C_{0}\left(z_{1}, z_{2}, r\right)
\end{aligned}
$$

Again, the brackets indicate how the bending rigidity may expressed as the sum of two separate expressions. This division is constructed such that the separate contributions both reduce to the DFT expressions in Appendix A, but, again, a term could be added and subtracted that has the property that it is zero when a mean-field approximation is made.

\section{APPENDIX D: PROPERTIES OF DIRECT CORRELATION FUNCTION}

In this appendix, we investigate some properties of the direct correlation function of the planar interface, $C_{0}\left(z_{1}, z_{2}\right.$, $r$ ), which turn out to be useful in rewriting the expression for the Tolman length in Appendix B.

In a uniform external field, the second Yvon equation in Eq. (15) reduces to

$$
0=k_{\mathrm{B}} T \int d \vec{r}_{12} \rho_{0}^{\prime}\left(z_{2}\right) C_{0}\left(z_{1}, z_{2}, r\right)
$$

The second Yvon equation in Eq. (13) applied to the situation in which a planar interface is curved by shifting the chemical potential off-coexistence leads to

$$
\mu_{1}=k_{\mathrm{B}} T \int d \vec{r}_{12}\left[\rho_{1}\left(z_{2}\right)+\frac{r^{2}}{2}\left(1-s^{2}\right) \rho_{0}^{\prime}\left(z_{2}\right)\right] C_{0}\left(z_{1}, z_{2}, r\right) .
$$

Eq. (D1) multiplied by $z_{1} \rho_{1}\left(z_{1}\right)$ and integrated over $z_{1}$ gives

$$
0=k_{\mathrm{B}} T \int_{-\infty}^{\infty} d z_{1} \int d \vec{r}_{12}\left(z_{1}+r s\right) \rho_{0}^{\prime}\left(z_{1}\right) \rho_{1}\left(z_{2}\right) C_{0}\left(z_{1}, z_{2}, r\right) .
$$

Eq. (D2) multiplied by $z_{1} \rho_{0}^{\prime}\left(z_{1}\right)$ and integrated over $z_{1}$ gives

$$
\begin{aligned}
& \int_{-\infty}^{\infty} d z_{1} \int d \vec{r}_{12} r s \rho_{0}^{\prime}\left(z_{1}\right) \rho_{1}\left(z_{2}\right) C_{0}\left(z_{1}, z_{2}, r\right) \\
& \quad=\frac{1}{2} \int_{-\infty}^{\infty} d z_{1} \int d \vec{r}_{12} r^{2}\left(1-s^{2}\right) z_{1} \rho_{0}^{\prime}\left(z_{1}\right) \rho_{0}^{\prime}\left(z_{2}\right) C_{0}\left(z_{1}, z_{2}, r\right),
\end{aligned}
$$

where Eq. (D3) has been used.
${ }^{1}$ J. S. Rowlinson and B. Widom, Molecular Theory of Capillarity (Clarendon, Oxford, 1982).

${ }^{2}$ J. D. van der Waals, Verh. K. Akad. Wet. Amsterdam, Sect. 1 8, 1 (1893); [J. Stat. Phys. 20, 200 (1979)].

${ }^{3}$ D. E. Sullivan, Phys. Rev. B 20, 3991 (1979).

${ }^{4}$ R. Evans, Adv. Phys. 28, 143 (1979).

${ }^{5}$ R. Evans, in Liquids at Interfaces, Les Houches XLVIII (1988), edited by J. Charvolin, J. F. Joanny, and J. Zinn-Justin (North-Holland, Amsterdam, 1990).

${ }^{6}$ P. Tarazona and R. Evans, Mol. Phys. 52, 847 (1984).

${ }^{7}$ J. G. Kirkwood and F. P. Buff, J. Chem. Phys. 17, 338 (1949).

${ }^{8}$ M. P. Allen and D. J. Tildesley, Computer Simulation of Liquids (Oxford University Press, Oxford, 1987).

${ }^{9}$ D. Frenkel and B. Smit, Understanding Molecular Simulation (Academic Press, San Diego, 1996).

${ }^{10} \mathrm{~J}$. Yvon, in Proceedings of the IUPAP Symposium on Thermodynamics, Brussels, 1948.

${ }^{11}$ T. G. Triezenberg and R. Zwanzig, Phys. Rev. Lett. 28, 1183 (1972).

${ }^{12}$ L. S. Ornstein and F. Zernike, Proc. Acad. Sci. Amsterdam 17, 793 (1914).

${ }^{13}$ R. C. Tolman, J. Chem. Phys. 17, 333 (1949).

${ }^{14}$ W. Helfrich, Z. Naturforsch. C 28, 693 (1973).

${ }^{15}$ E. M. Blokhuis and D. Bedeaux, Physica A 184, 42 (1992).

${ }^{16}$ E. M. Blokhuis and D. Bedeaux, Mol. Phys. 80, 705 (1993).

${ }^{17}$ A. E. van Giessen, E. M. Blokhuis, and D. J. Bukman, J. Chem. Phys. 108, 1148 (1998).

${ }^{18}$ S. J. Hemingway, J. R. Henderson, and J. S. Rowlinson, Faraday Symp. Chem. Soc. 16, 33 (1981).

${ }^{19}$ J. R. Henderson and P. Schofield, Proc. R. Soc. London, Ser. A 380, 211 (1982).

${ }^{20}$ P. Schofield and J. R. Henderson, Proc. R. Soc. London, Ser. A 379, 231 (1982).

${ }^{21}$ R. Lovett, P. W. DeHaven, J. J. Vieceli, and F. P. Buff, J. Chem. Phys. 58, 1880 (1973).

${ }^{22}$ E. M. Blokhuis, J. Groenewold, and D. Bedeaux, Mol. Phys. 96, 397 (1999).

${ }^{23}$ E. M. Blokhuis, J. Chem. Phys. 130, 014706 (2009).

${ }^{24}$ E. M. Blokhuis and A. E. van Giessen, J. Phys.: Condens. Matter 25, 225003 (2013)

${ }^{25}$ A. O. Parry and C. J. Boulter, J. Phys.: Condens. Matter 6, 7199 (1994).

${ }^{26}$ J. Meunier, J. Phys. 48, 1819 (1987).

${ }^{27}$ O.-Y. Zhong-can and W. Helfrich, Phys. Rev. A 39, 5280 (1989).

${ }^{28}$ J. W. Gibbs, Collected Works (Dover, New York, 1961).

${ }^{29}$ J. C. Barrett, J. Chem. Phys. 111, 5938 (1999).

${ }^{30}$ J. D. Weeks, J. Chem. Phys. 67, 3106 (1977).

31 J. D. Weeks and W. van Saarloos, J. Phys. Chem. 93, 6969 (1989).

${ }^{32}$ J. D. Weeks, W. van Saarloos, D. Bedeaux, and E. M. Blokhuis, J. Chem. Phys. 91, 6494 (1989).

${ }^{33}$ M. H. Waldor and D. E. Wolf, J. Chem. Phys. 85, 6082 (1986).

${ }^{34}$ M. P. A. Fisher and M. Wortis, Phys. Rev. B 29, 6252 (1984).

${ }^{35}$ G. Gompper and S. Zschocke, Phys. Rev. A 46, 4836 (1992).

${ }^{36}$ E. M. Blokhuis and J. Kuipers, J. Chem. Phys. 124, 074701 (2006).

${ }^{37}$ Y. Rosenfeld, Phys. Rev. Lett. 63, 980 (1989); R. Roth, J. Phys. Condens. Matter 22, 063102 (2010). 